\title{
Classifications in Brief: Lauge-Hansen Classification of Ankle Fractures
}

\author{
Jason P. Tartaglione MD, Andrew J. Rosenbaum MD, Mostafa Abousayed MD, \\ John A. DiPreta MD
}

Received: 7 January 2015/Accepted: 9 April 2015/Published online: 22 April 2015

(C) The Association of Bone and Joint Surgeons \& 2015

\section{History}

Ankle fractures are common musculoskeletal injuries that occur in a bimodal distribution, with peaks in younger men and older women [2], the former related to high-energy trauma and the latter to osteopenia and osteoporosis. Although ankle fractures currently account for 9\% of fractures, incidence and severity are increasing [1]. This is attributed to the increased life expectancy among older individuals and improved survival of patients with severe foot and ankle trauma [23, 26].

The first ankle fracture classification, credited to Percival Pott, described three types of ankle fractures based on the number of malleoli involved: unimalleolar, bimalleolar, and trimalleolar [21]. Despite its ease of use and reproducibility, the classification did not effectively guide management, as it failed to differentiate stable from unstable injuries.

This was the impetus for the work of Niel Lauge-Hansen (1899-1976), a Danish physician who studied ankle fractures during the 1940s and 1950s, ultimately creating a classification system based on a rotational mechanism of

Each author certifies that he or she has no commercial association (eg, consultancies, stock ownership, equity interest, patent/licensing arrangements, etc) that might pose a conflict of interest in connection with the submitted article.

All ICMJE Conflict of Interest Forms for authors and Clinical Orthopaedics and Related Research ${ }^{\mathbb{R}}$ editors and board members are on file with the publication and can be viewed on request.

J. P. Tartaglione ( $\square)$, A. J. Rosenbaum, M. Abousayed, J. A. DiPreta

Division of Orthopaedics, Albany Medical Center, 1367

Washington Avenue, Suite 202, Albany, NY 12206, USA

e-mail: jptartag@gmail.com injury [11-15]. Lauge-Hansen's classification, which was published in a 1950 issue of Archives of Surgery, has become one of the most widely used ankle fracture classification systems [12]. Although still considered a landmark work regarding the biomechanics and deforming forces of ankle fractures, the quality, validity, and reproducibility of the Lauge-Hansen classification have been challenged [17, 19, 22, 25].

\section{Purpose}

An ideal fracture classification would be reproducible, widely recognized, relevant for prognosis, and useful in terms of clinical decision-making, documentation, and research.

The primary normal motion of the ankle is dorsiflexion and plantar flexion, with osseous anatomy and ligamentous complexes that provide stability in all planes and axes of rotation. When these structures are injured, there is substantial risk of instability. Appropriate reconstruction therefore is important, and a classification scheme that identifies injury patterns and guides treatment would be desirable.

\section{Description of the Lauge-Hansen System}

Niel Lauge-Hansen used freshly amputated limbs to develop an ankle fracture classification based on foot position at the time of the traumatic event (supination or pronation) and the direction of the deforming forces (abduction, adduction, or external rotation) [12].

Cadaveric tibias were fixed with a vice and nails while a rotational deforming force was applied by hand with the 
Table 1. Categories and stages of the Lauge-Hansen classification system

\begin{tabular}{ll}
\hline Category & Stage \\
\hline Supination external rotation & 1 Injury of the anterior inferior tibiofibular ligament \\
& 2 Oblique/spiral fracture of the distal fibula \\
& 3 Injury of the posterior inferior tibiofibular ligament or avulsion of the posterior malleolus \\
& 4 Medial malleolus fracture or injury to the deltoid ligament \\
& 1 Transverse fracture of the distal fibula \\
& 2 Vertical fracture of the medial malleolus \\
Supination adduction & 1 Medial malleolus fracture or injury to the deltoid ligament \\
& 2 Injury of the anterior inferior tibiofibular ligament \\
& 3 Oblique/spiral fracture of the fibula proximal to the tibial plafond \\
Pronation external rotation & 4 Injury of the posterior inferior tibiofibular ligament or avulsion of the posterior malleolus \\
& 1 Medial malleolus fracture or injury to the deltoid ligament \\
& 2 Injury of the anterior inferior tibiofibular ligament \\
& 3 Transverse or comminuted fracture of the fibula proximal to the tibial plafond \\
Pronation abduction &
\end{tabular}

foot in a supinated or pronated position. Supination was comprised of internal rotation of the ankle about the tibial axis, forefoot adduction, and hindfoot inversion; pronation was made up of external rotation of the ankle about the tibial axis, forefoot abduction, and hindfoot eversion. For each combination of foot position and deforming force, a consistently reproducible fracture pattern was elucidated. Lauge-Hansen created four categories and 13 subgroups of ankle fractures based on his study, which used between four and eight experiments in each of the categories described (Table 1) [12].

Supination external rotation injuries, the most commonly occurring of Lauge-Hansen's categories, were produced in seven experiments [8, 12]. In Stage 1, the anterior inferior tibiofibular ligament (AITFL) was injured. Subsequently, in Stage 2, an oblique/spiral fracture of the distal fibula occurred. With more force, the posterior inferior tibiofibular ligament (PITFL) was injured or the posterior malleolus was fractured in Stage 3. Finally, in Stage 4 , the medial malleolus was fractured or the deltoid ligament was injured [12].

Supination adduction injuries occurred in eight experiments and consisted of two stages. In Stage 1, either a transverse fracture of the lateral malleolus below the level of the tibial plafond was produced or the lateral ligaments of the ankle were avulsed off of the distal fibula. In Stage 2, either a vertical medial malleolus fracture or injury to the deltoid ligament occurred [12].

Pronation external rotation injuries were recreated in four experiments, with either deltoid ligament rupture or avulsion fractures of the medial malleolus evident in Stage 1. Injuries of the AITFL were seen in Stage 2, oblique/ spiral fibula fractures proximal to the level of the tibial plafond were observed in Stage 3, and fractures of the posterior malleolus or injury to the PITFL ensued in Stage 4 [12].

Pronation abduction injuries were evaluated in three experiments. Stage 1 injuries involved fractures of the medial malleolus, whereas Stage 2 injuries were associated with damage to the AITFL. In Stage 3, transverse or comminuted fibula fractures were observed above the level of the tibial plafond [12].

In an attempt to create a classification system that did not rely on the mechanism of injury, which rarely is truly known and often speculated, Danis and Weber created an ankle fracture classification based on radiographic criteria. This classification system took into consideration the position of the distal fibular fracture in relation to the syndesmosis [4, 27]. According to the Danis-Weber classification, a Type A fracture occurs below the level of the tibial plafond and may be associated with oblique or vertical medial malleolar fractures [4, 27], which correlates with the supination adduction pattern described by LaugeHansen (Fig. 1) [12]. Per Danis-Weber, Type B fractures originate at the level of the tibial plafond and extend proximally in an oblique fashion [4, 27] (correlating with Lauge-Hansen's supination external rotation injuries [Fig. 2]), and may be accompanied by a medial malleolus fracture or deltoid ligament rupture [12]. Danis-Weber Type $\mathrm{C}$ fractures occur proximal to the level of the tibial plafond and often have an associated syndesmotic injury [4, 12, 27], which correlates with pronation external rotation (Fig. 3) and pronation abduction patterns (Fig. 4) described by Lauge-Hansen. Danis-Weber Type C fractures can be associated with medial malleolus fractures or injury to the deltoid ligament.

Finally, expanding on the work of Danis and Weber, the AO (Arbeitsgemeinschaft für Osteosynthesefragen, known 


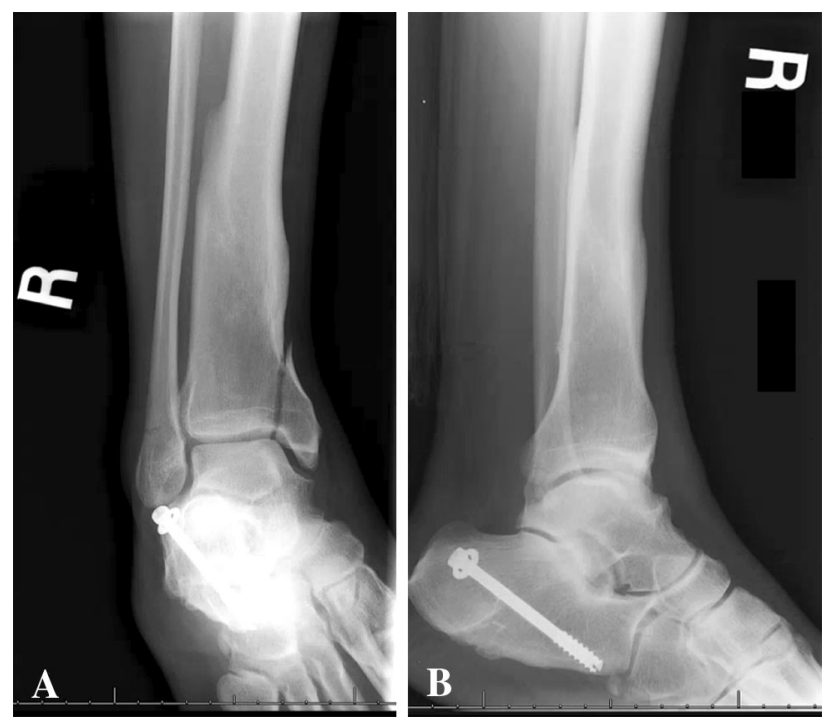

Fig. 1A-B (A) Mortise and (B) lateral plain radiographs of a right ankle show the classic appearance of a Lauge-Hansen supination adduction injury, which corresponds to the Danis-Weber Type A and AO/OTA 44-A fracture patterns.
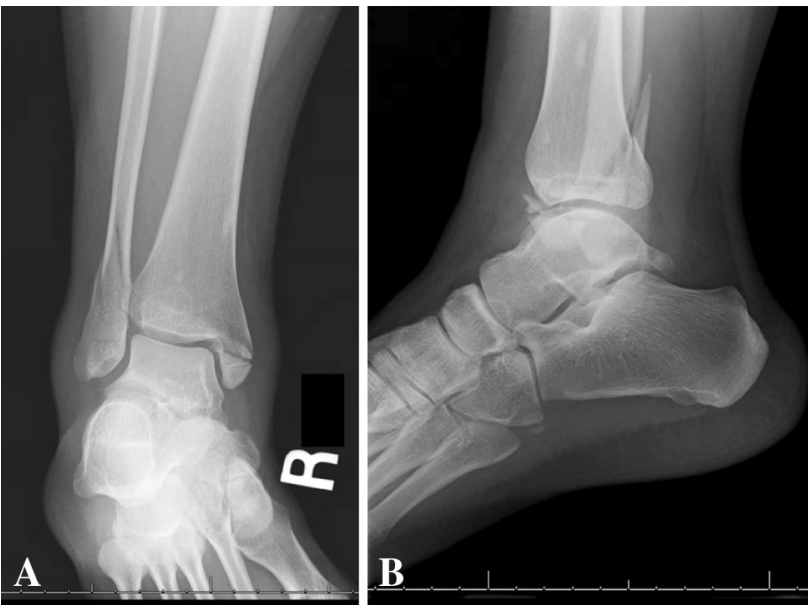

Fig. 2A-B (A) Mortise and (B) lateral plain radiographs of a right ankle show the classic appearance of a Lauge-Hansen supination external rotation injury, which corresponds to the Danis-Weber Type $\mathrm{B}$ and AO/OTA 44-B fracture patterns.

in English as the "Association for the Study of Internal Fixation")/Orthopaedic Trauma Association (OTA) system classifies all long bone fractures with a systematic approach, based on location, topography, and extent of bony lesion. Like the Danis-Weber classification, it is based only on radiographic criteria, in contrast to the Lauge-Hansen system, which unlike all other appendicular fracture classification systems, incorporates the mechanism of injury. In the AO/OTA classification, ankle fractures are classified as infrasyndesmotic (correlating to
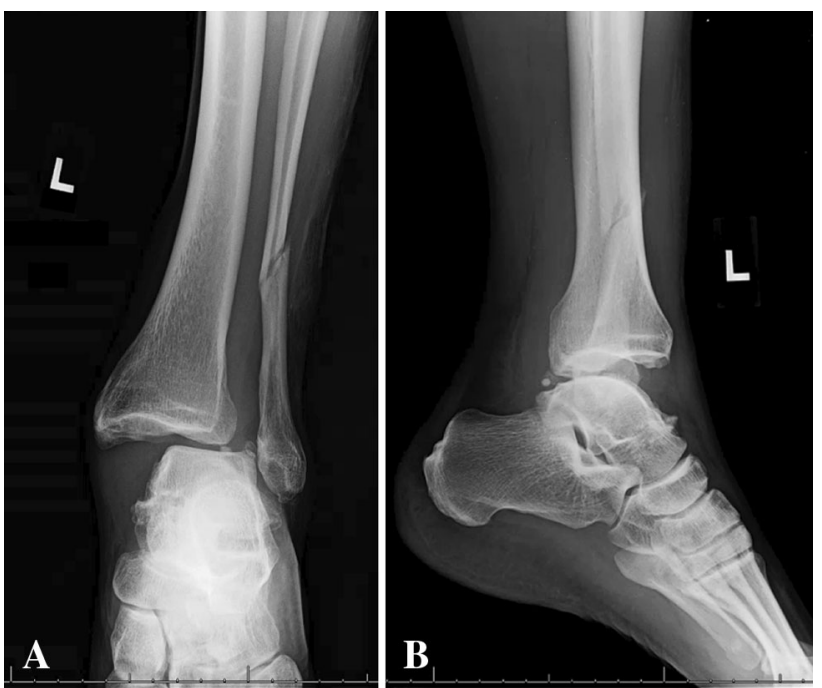

Fig. 3A-B (A) AP and (B) lateral plain radiographs of a left ankle show the classic appearance of a Lauge-Hansen pronation external rotation injury, which corresponds to the Danis-Weber Type $\mathrm{C}$ and AO/OTA 44-C fracture patterns.
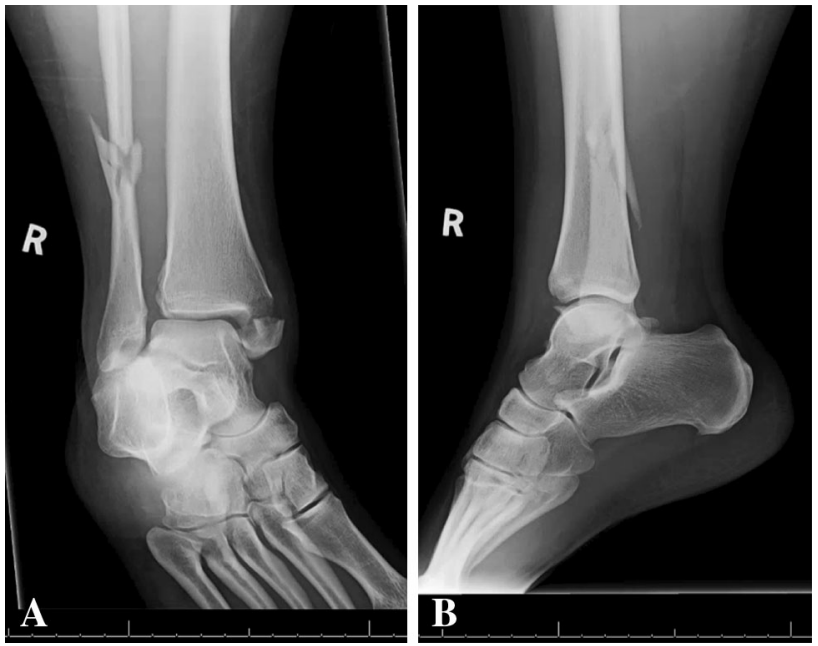

Fig. 4A-B (A) Mortise and (B) lateral plain radiographs of a right ankle show the classic appearance of a Lauge-Hansen pronation abduction injury, which corresponds to the Danis-Weber Type $\mathrm{C}$ and AO/OTA 44-C fracture patterns.

Danis-Weber Type A injuries), transsyndesmotic (correlating to Danis-Weber Type B injuries) or suprasyndesmotic (correlating to Danis-Weber Type C injuries) with further subsets based on presence or absence of medial or posterior malleolar injuries [18]. This is in contrast to the Danis-Weber classification that completely ignores the medial and posterior structures. Additionally, the AO/OTA classification system encompasses the fracture patterns described by Lauge-Hansen and DanisWeber (Table 2). 
Table 2. Comparison of the Danis-Weber, Lauge-Hansen, and AO/OTA classification systems

\begin{tabular}{|c|c|c|c|}
\hline Fibular fracture location & Danis-Weber classification & Lauge-Hansen classification & $\begin{array}{l}\text { AO/OTA classification of tibial malleolar } \\
\text { fractures }\end{array}$ \\
\hline Infrasyndesmotic & Type A & SAD I, II & $\begin{array}{l}\text { 44-A1 (isolated lateral) } \\
\text { 44-A2 (lateral and medial) } \\
\text { 44-A3 (lateral, medial, and posterior) }\end{array}$ \\
\hline Transsyndesmotic & Type B & SER I, II, III, IV & $\begin{array}{l}\text { 44-B1 (isolated lateral) } \\
\text { 44-B2 (lateral and medial) } \\
\text { 44-B3 (lateral, medial, and Volkmann's fracture) }\end{array}$ \\
\hline Suprasyndesmotic & Type C & PER I, II, III, IV; PA I, II, III & $\begin{array}{l}\text { 44-C1 (simple diaphyseal) } \\
\text { 44-C2 (multifragmentary) } \\
\text { 44-C3 (proximal) }\end{array}$ \\
\hline
\end{tabular}

OTA = Orthopaedic Trauma Association; SAD = supination adduction; SER = supination external rotation; PER = pronation external rotation; $\mathrm{PA}=$ pronation abduction .

\section{Validation}

In 1980, Yde advocated use of the Lauge-Hansen's classification system, as he was able to successfully classify 488 ankle fractures based on Lauge-Hansen's categories and stages [28]. Despite Yde's work, multiple studies have since challenged the validity of the Lauge-Hansen classification system [5, 6, 17, 19, 22, 25].

Interobserver and intraobserver reliabilities are fair at best for the Lauge-Hansen classification. Nielsen et al. [19] had four observers evaluate and classify the radiographs of ankle fractures in 118 patients using Lauge-Hansen's classification. Fifty-one of $118(43 \%)$ radiographs were classified identically by all four observers. To determine intraobserver variation, the same four observers classified the same radiographs 6 weeks after their initial classification and found that their first and second classifications were identical in 75 to 97 of the 118 radiographs $(64 \%-$ $82 \%)$ [19].

Later investigators have been unable to duplicate the mechanical events or injuries described by Lauge-Hansen. Using modern biomechanical techniques, Michelson et al. [17] were unable to reproduce the supination external rotation fracture pattern in 32 cadaveric ankles using LaugeHansen's proposed method. Gardner et al. [5] studied 49 ankle fractures that fit into Lauge-Hansen's classification and compared plain radiographs with MR images. They showed that the proposed soft tissue injuries associated with the different Lauge-Hansen categories and stages were not accurately predicted [5]. In another investigation by Haraguchi and Armiger [6], the mechanism by which the distal fibula breaks in supination external rotation injuries was questioned, as the authors were able to produce a short oblique fracture of the distal fibula with the foot in the pronated position. Despite contradicting Lauge-Hansen's system, this makes sense. As the foot is externally rotated from the supinated position (internal rotation of the ankle, hindfoot inversion, and forefoot adduction), it inevitably is placed in pronation (external rotation of the ankle, hindfoot eversion, and forefoot abduction). This also is shown because supination external rotation injuries often involve disruption of the deltoid ligament, which in a supinated foot would be under compression and unlikely to tear. In addition, Haraguchi and Armiger [6] reported that suprasyndesmotic fibular fractures can occur when increasing the abduction moment at the ankle, thus highlighting the importance of applied loads; namely, the combination of external rotation and abduction.

Several investigators have evaluated the validity of the Danis-Weber and AO/OTA classification systems [3, 16]. Malek et al. [16] assessed the reproducibility and reliability of the Danis-Weber classification system. Five observers classified 50 sets of blinded radiographs and then again after an interval of 4 weeks. For interobserver agreement, the mean kappa value was 0.61 and the proportion of agreement was $78 \%$, and for intraobserver agreement the mean kappa value was 0.74 with an $85 \%$ observed agreement [16]. Using similar techniques and statistics, Craig and Dirschl [3] had six reviewers evaluate 50 radiographs of ankle fractures and found the mean kappa value for interobserver reliability of the AO/OTA classification to be 0.61 .

\section{Limitations}

Lauge-Hansen's original technique required manipulations and application of forces by hand to a fixed foot [12]. Such methodology is not only imprecise but also fails to accurately recreate the in vivo forces experienced by a patient while sustaining an ankle fracture; namely the combination of axial load and rotational forces as the body moves 
relative to a foot planted on the ground. In an attempt to account for these limitations, Kwon et al. [10] and Rodriguez et al. [24] studied YouTube videos and correlated mechanisms of injury with radiographic findings. Rodriguez et al. [24] found that when injury video clips were matched to their corresponding radiographs, the LaugeHansen classification system had a 65\% (17 of 26 ankle fractures) consistency rate in predicting fracture patterns from the deforming-injury mechanism. Using the same techniques, they determined that the AO/OTA classification system had a greater consistency rate of $81 \%$ (21 of 26 ankle fractures) [24].

A classification system should be comprehensive and have the capacity to describe all the types of fractures in its range of classification. Studies have shown that a small percentage of ankle fractures, such as isolated fractures of the posterior tibial margin, do not fit into the Lauge-Hansen classification [5, 28]. The AO/OTA classification does not have this limitation because it is a comprehensive classification of all long bone fractures including those of the tibial plafond and both malleoli.

The poor interobserver reliability of the Lauge-Hansen, Danis-Weber, and AO/OTA classifications indicates that orthopaedic surgeons interpret these classifications differently, suggesting these systems are an ineffective means for physician-to-physician communication [3, 16, 19, 22, 25]. One of the keys to the Lauge-Hansen classification is the geometry of the medial malleolus fracture. Transverse fractures are thought to be a product of pronation patterns of injury and vertical fractures are thought to represent supination injury patterns. However, many medial malleolus fractures are oblique, which is a major source of error and confusion, conceivably resulting in high interobserver variation. The Danis-Weber classification appears to be an easier classification system for physician-tophysician communication owing to its simplicity; however, the Danis-Weber system completely overlooks the medial side of the ankle and has been shown to be a predictor of functional and radiographic outcomes only for unimalleolar ankle fractures [9]. The AO/OTA classification built on the Danis-Weber classification by taking into account injuries of the medial and posterior malleoli.

Originally, the Lauge-Hansen classification system was created to direct closed reduction. The reduction maneuver could be devised by putting the foot and ankle back in the original position and by reversing the mechanism of injury. Once a major advantage of Lauge-Hansen's system, this concept has become less important in guiding treatment as operative fixation becomes more popular.

Unstable ankle fractures have better overall clinical, radiographic, and arthritis scores when treated surgically [20, 29]. Additionally, ankle fractures that are poorly reduced result in worse overall patient outcomes than fractures with a well-aligned ankle mortise [9]. Therefore, to guide treatment, an ankle fracture classification system should reliably predict fracture pattern stability, which is determined by the complex osseous and ligamentous anatomy of the ankle. Comparing radiographs with corresponding MR images of 51 ankle fractures, Hermans et al. [7] reported that the Lauge-Hansen classification reliably predicted injury to the syndesmosis. Contrary to the findings of Hermans et al., using similar methodology, Gardner et al. [5] reported that 53\% (26 of 49 ankle fractures) of the time, the Lauge-Hansen classification system did not reliably predict ligamentous injuries associated with its categories and stages of classification. This is a substantial limitation of the Lauge-Hansen classification because it fails to characterize the integrity of the osseous and ligamentous structures surrounding the ankle, which are vital to stability. Therefore, it is not yet well understood how much clinical guidance the Lauge-Hansen system offers.

Although Lauge-Hansen's work is valuable, it falls short on numerous points. As stated before, the Danis-Weber system is based on fracture pattern that is determined from radiographic appearance and is not based on deduced and inaccurate concepts like foot position and mechanism of injury. However, this system ignores the medial side of the ankle, which plays an important role in determining ankle stability and function.

Neither the Lauge-Hansen nor the Danis-Weber systems were developed for use in contemporary open fracture care, therefore they have substantial limitations. The AO/OTA system, by contrast is superior to the Lauge-Hansen and Danis-Weber systems in many respects. Like the DanisWeber system, the AO/OTA classification does not rely on mechanism of injury or foot position and is determined from radiographs. Because it is part of a scheme which covers all parts of the body, the AO/OTA classification also is able to characterize fractures not categorized by the Lauge-Hansen and Danis-Weber systems, including distal tibial plafond fractures. Finally, the AO/OTA classification system also has the advantage of identifying all four basic patterns described by Lauge-Hansen (Table 2). Therefore, the understanding acquired from Lauge-Hansen can be incorporated in the treatment of ankle fractures when using the AO/OTA classification.

\section{Conclusions and Uses}

The Lauge-Hansen classification system was developed based on a cadaveric study which included imprecise replication of the true pathobiomechanics experienced during ankle fracture and incorporated deduced concepts like foot position at the time of injury and direction of the deforming force. The AO/OTA classification, which is a 
comprehensive classification system of long bones that classifies fractures based on fracture pattern, has a multitude of advantages over the Lauge-Hansen system. Despite its limitations, the Lauge-Hansen classification system continues to be one of the most widely used and accepted ankle fracture classification systems, and has enhanced our understanding of ankle fractures. However, it is essential that future studies continue to investigate mechanism of injury, fracture patterns, and optimal treatments for ankle fractures.

\section{References}

1. Court-Brown CM, Caesar B. Epidemiology of adult fractures: a review. Injury. 2006;37:691-697.

2. Court-Brown CM, McBirnie J, Wilson G. Adult ankle fractures: an increasing problem? Acta Orthop Scand. 1998;69:43-47.

3. Craig WL 3rd, Dirschl DR. Effects of binary decision making on the classification of fractures of the ankle. J Orthop Trauma. 1998;12:280-283.

4. Danis R. Les fractures malleolaires. In: Danis R (ed). Theorie et Pratique de l'Osteosynthese. Paris, France: Masson; 1949:133165.

5. Gardner MJ, Demetrakopoulos D, Briggs, SM, Helfet DL, Lorich DG. The ability of the Lauge-Hansen classification to predict ligament injury and mechanism in ankle fractures: an MRI study. J Orthop Trauma. 2006;20:267-272.

6. Haraguchi N, Armiger RS. A new interpretation of the mechanism of ankle fracture. J Bone Joint Surg Am. 2009;91:821-829.

7. Hermans JJ, Wentink N, Beumer A, Hop WC, Heijboer MP, Moonen AF, Ginai AZ. Correlation between radiological assessment of acute ankle fractures and syndesmotic injury on MRI. Skeletal Radiol. 2012;41:787-801.

8. Jensen SL, Andresen BK, Mencke S, Nielsen PT. Epidemiology of ankle fractures: a prospective population-based study of 212 cases in Aalborg, Denmark. Acta Orthop Scand. 1998;69:48-50.

9. Kennedy JG, Johnson SM, Collins AL, DalloVedova P, McManus WF, Hynes DM, Walsh MG, Stephens MM. An evaluation of the Weber classification of ankle fractures. Injury. 1998;29:577580 .

10. Kwon JY, Chacko AT, Kadzielski JJ, Appleton PT, Rodriguez EK. A novel methodology for the study of injury mechanism: ankle fracture analysis using injury videos posted on YouTube.com. J Orthop Trauma. 2010;24:477-482.

11. Lauge-Hansen N. Ligamentous ankle fractures: diagnosis and treatment. Acta Chir Scand. 1949;97:544-550.

12. Lauge-Hansen N. Fractures of the ankle: II. Combined experimentalsurgical and experimental-roentgenologic investigations. Arch Surg. 1950;60:957-985.
13. Lauge-Hansen N. Fractures of the ankle: IV. Clinical use of genetic roentgenologic diagnosis and genetic reduction. AMA Arch Surg. 1952;64:488-500.

14. Lauge-Hansen N. Fractures of the ankle: V. Pronation-dorsiflexion fracture. AMA Arch Surg. 1953;67:813-820.

15. Lauge-Hansen N. Fractures of the ankle: III. Genetic roentgenologic diagnosis of fractures of the ankle. Am J Roentgenol Radium Ther Nucl Med. 1954;71:456-471.

16. Malek IA, Machani B, Mevcha AM, Hyder NH. Inter-observer reliability and intra-observer reproducibility of the Weber classification of ankle fractures. J Bone Joint Surg Br. 2006;88:12041206.

17. Michelson J. Solocoff D. Waldman B, Kendell K, Ahn U. Ankle fractures: the Lauge-Hansen classification revisited. Clin Orthop Relat Res. 1997;345:198-205.

18. Muller ME, Nazarian S, Koch P, Schatzker J. Tibia/fibula. The Comprehensive Classification of Fractures of Long Bones. Berlin, Germany: Springer-Verlag; 1990:148-191.

19. Nielsen JO, Dons-Jensen H, Sorensen HT. Lauge-Hansen classification of malleolar fractures: an assessment of the reproducibility in 118 cases. Acta Orthop Scand. 1990;61:385387.

20. Phillips WA, Schwartz HS, Keller CS, Woodward HR, Rudd WS, Spiegel PG, Laros GS. A prospective, randomized study of the management of severe ankle fractures. J Bone Joint Surg Am. 1985;67:67-78.

21. Pott P. Some few general remarks on fractures and dislocations: 1758. Clin Orthop Relat Res. 2007;458:40-41.

22. Rasmussen S, Madsen PV, Bennicke K. Observer variation in the Lauge-Hansen classification of ankle fractures: precision improved by instruction. Acta Orthop Scand. 1993;64:693-694.

23. Richter M, Thermann H, Wippermann B, Otte D, Schratt HE, Tscherne H. Foot fractures in restrained front seat car occupants: a long-term study over twenty-three years. J Orthop Trauma. 2001;15:287-293.

24. Rodriguez EK, Kwon JY, Herder LM, Appleton PT. Correlation of $\mathrm{AO}$ and Lauge-Hansen classification systems for ankle fractures to the mechanism of injury. Foot Ankle Int. 2013;34:15161520.

25. Thomsen NO, Overgaard S, Olsen LH, Hansen H, Nielsen ST. Observer variation in the radiographic classification of ankle fractures. J Bone Joint Surg Br. 1991;73:676-678.

26. Toole WP, Elliott M, Hankins D, Rosenbaum C, Harris A, Perkins C. Are low-energy open ankle fractures in the elderly the new geriatric hip fracture? J Foot Ankle Surg. 2015;54:203-206.

27. Weber BG. Die Verletzungen des oberen Sprunggelenkes. 2nd ed. Berne, Switzerland: Verlag Hans Huber; 1972.

28. Yde J. The Laugen Hansen classification of malleolar fractures. Acta Orthop Scand. 1980;51:181-192.

29. Yde J, Kristensen KD. Ankle fractures: supination-eversion fractures of stage IV. Primary and late results of the operative and non-operative treatment. Acta Orthop Scand. 1980;51:981-990. 